\title{
Verapamil Favorably Influences Hepatic Microvascular Exchange and Function in Rats with Cirrhosis of the Liver
}

\author{
J. Reichen and M. Le \\ Department of Clinical Pharmacology, University of Berne, CH-3010 Berne, Switzerland; Division of Gastroenterology, \\ Department of Medicine, University of Colorado School of Medicine, Denver, Colorado 80262
}

\begin{abstract}
The effect of the calcium channel blocking agent, verapamil, on microcirculatory patterns and hepatic function was investigated in the perfused liver of cirrhotic rats. Compared with controls, cirrhotic livers had higher vascular resistance, increased intrahepatic shunting, and smaller extravascular albumin space and larger extravascular sucrose space, as determined by a multipleindicator dilution technique. Hepatic function, estimated by determining propranolol and antipyrine extraction, was markedly reduced in cirrhotic livers. Portal pressure was then reduced $25 \%$ either pharmacologically by verapamil or hydrodynamically by lowering inflow. Verapamil decreased vascular resistance by $22 \%$. This was associated with a $38 \%$ reduction in intrahepatic shunting and a $62 \%$ increase in extravascular albumin space. Hydrodynamically lowering pressure had no or adverse effects. The verapamil-induced improvement in microcirculatory characteristics was associated with a significant improvement in oxygen consumption $(+21 \%)$ and antipyrine clearance $(+20 \%)$. We conclude that the microvascular distortions of liver cirrhosis in the rat are partially reversible by vasodilators like verapamil.
\end{abstract}

\section{Introduction}

Pharmacotherapy of portal hypertension has been limited so far to lowering portal pressure by decreasing splanchnic blood flow, either acutely with vasopressin or somatostatin (1) or chronically with beta blockers such as propranolol $(2,3)$. Decreasing perfusion of an organ with already compromised function could lead to a further decrease in function and eventually to decompensation.

An alternative approach, namely lowering pressure by decreasing hepatic resistance, has not yet been systematically explored. It is unknown to what degree, if any, portal hypertension and microvascular alterations of cirrhosis, such as intrahepatic shunting and sinusoidal capillarization (4-7), are reversible. This approach appears promising, however, because one would expect maintenance or even improvement of hepatic function when hepatic blood flow is maintained.

We therefore investigated the effect of a calcium channel blocker, verapamil, on hepatic microcirculation and function in the perfused liver of cirrhotic rats. We have investigated ver-

This study has been presented in part at the 1984 meeting of the AASLD and was published as an abstract (1984. Hepatology. 4:1027). 1986

Received for publication 1 July 1985 and in revised form 7 April

J. Clin. Invest.

(c) The American Society for Clinical Investigation, Inc.

0021-9738/86/08/0448/08 \$1.00

Volume 78, August 1986, 448-455 apamil because $(a)$ it has a high first-pass effect (8), (b) calcium channel blockers affect all smooth muscle cells examined (9), and $(c)$ verapamil has been shown to increase estimated hepatic blood flow in humans (10). Verapamil administered intravenously has recently been shown to reduce wedged hepatic vein pressure in humans (11). Finally, we have presented preliminary evidence that the drug improves indocyanine green clearance in patients with liver cirrhosis (12).

\section{Methods}

Materials. Male Sprague-Dawley rats were obtained from Charles River Breeding Laboratories, Wilmington, MA. ${ }^{57} \mathrm{Co}$ microspheres, diam $15 \pm 1$ $\mu \mathrm{m}$ (sp act $10 \mathrm{mCi} / \mathrm{g}$ ), $\left[{ }^{14} \mathrm{C}\right]$ sucrose (sp act $673 \mathrm{mCi} / \mathrm{mmol}$ ), and $\left[{ }^{14} \mathrm{C}\right]$ antipyrine (sp act $59 \mathrm{mCi} / \mathrm{mmol}$ ) were obtained from New England Nuclear, Boston, MA. $\left[{ }^{3} \mathrm{H}\right.$ ]propranolol (sp act $16.6 \mathrm{Ci} / \mathrm{mmol}$ ) was purchased from Amersham Corp., Arlington Heights, IL. ${ }^{51} \mathrm{Cr}$-erythrocytes were prepared by incubating $1 \mathrm{ml}$ of human erythrocytes with $0.1 \mathrm{mCi}$ of $\mathrm{Na}^{5}{ }^{51} \mathrm{Cr}_{2} \mathrm{O}_{4}$ (New England Nuclear). ${ }^{125} \mathrm{I}$-albumin was prepared by the lactate peroxidase method. Free iodine was removed by chromatography on a 10-ml Sephadex G-100 column and by dialysis overnight against phosphate-buffered saline. The final product had a specific activity of $1,200 \mathrm{cpm} / \mu \mathrm{g}$ protein and was $>95 \%$ precipitable by trichloroacetic acid. Verapamil $\mathrm{HCl}$ was obtained commercially in injectable form.

Induction of cirrhosis. Cirrhosis was induced in rats, weighing 125$150 \mathrm{~g}$ at the start of the study, by chronic exposure to phenobarbital and carbon tetrachloride according to McLean et al. (13). Treatment was ceased at least $2 \mathrm{wk}$ prior to the study. Control animals were kept under identical conditions but exposed to neither toxin. Treated rats gained less weight than control rats, their weights at the time of study averaging $464 \pm 48$ and $486 \pm 59 \mathrm{~g}$, respectively. The corresponding liver weights were $20.6 \pm 3.5$ and $18.7 \pm 2.0 \mathrm{~g}$. All animals had cirrhosis by gross inspection and histologic examination. $73 \%$ of the animals had ascites $(>2$ $\mathrm{ml}$ ) at the time of study.

Rat liver perfusion. Animals were anesthetized with pentobarbital 50 $\mathrm{mg} / \mathrm{kg}$ intraperitoneally and were heparinized $(1,000 \mathrm{IU} / \mathrm{kg})$ prior to cannulation of the portal vein. Portal pressure was measured in vivo by cannulating the ileocolic vein with PE 50 tubing (Clay-Adams, Parsipanny, NJ). Portal perfusion was then performed as previously described in detail $(14,15)$. A semisynthetic medium consisting of washed human erythrocytes ( $20 \% \mathrm{vol} / \mathrm{vol})$, bovine serum albumin ( $2 \% \mathrm{wt} / \mathrm{vol})$, and dextrose $(0.1 \% \mathrm{wt} / \mathrm{vol})$ in Krebs-Ringer bicarbonate buffer was used. Portal perfusing pressure was set at the value measured in vivo using a constriction valve in the portal inflow (see Fig. 1). The perfusion system employed a hydrostatic head with a constricting valve (No. 2 in Fig. 1) permitting regulation of inflow. This setup permits detection of alterations in pressure by the in-line hydrostatic manometer (No. 3 in Fig. 1). Alterations in pressure $(p)$ and/or flow reflect alterations in resistance of the liver according to

$R=\Delta p / F$

where $\Delta p$ is the pressure gradient across the organ, and $F$ is blood flow. $\Delta p$ was set equal to portal pressure in that hepatic vein pressure was assumed to be zero by placing the hepatic vein catheter (No. 5 in Fig. 1) below the hepatic hilum. This assumption was verified in some experiments by placing a manometer into the hepatic vein cannula. Viability 


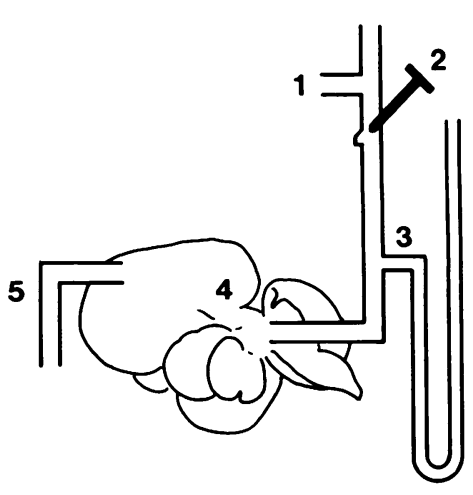

Figure 1. Schematic representation of the perfusion system. Inflow towards the liver can be regulated by a valve (2). Excess blood delivered reaches the reservoir via a bypass $(I)$. Pressure is measured by a manometer (3) in line with the portal vein cannula (4). Hepatic vein pressure equals zero because the efflux catheter is placed below the liver hilum (5).

of the perfused organ was assessed by measurement of portal flow, oxygen consumption, and transaminase and potassium release into the medium as previously described (15).

Experimental design. After an equilibration period of $20 \mathrm{~min}$, multiple indicator dilution studies were performed. ${ }^{51} \mathrm{Cr}$-labeled erythrocytes ( 5 $\mu \mathrm{Ci}),{ }^{125} \mathrm{I}$-albumin $(10 \mu \mathrm{Ci})$, and $\left[{ }^{14} \mathrm{C}\right]$ sucrose $(0.5 \mu \mathrm{Ci})$ were rapidly injected into the portal vein. Total hepatic venous outflow was collected at 1 -s intervals for $30 \mathrm{~s}$. Radioactivity of the $\gamma$-emitters $\left({ }^{51} \mathrm{Cr},{ }^{57} \mathrm{Co}\right.$, and ${ }^{125} \mathrm{I}$ ) was counted in a Packard Autogammaspectrometer (Packard Instrument Co., Inc., Downers Grove, IL) with appropriate correction for crossover between the different radioisotopes. ${ }^{14} \mathrm{C}$ - and ${ }^{3} \mathrm{H}$-radioactivity was counted on a Packard 460CD liquid scintillation counter using Budgetsolve as scintillator. External standardization was used to correct for quenching.

Shunt fraction was determined by injection of $40,000-50,000{ }^{57} \mathrm{Co}$ microspheres (diam $15 \pm 1 \mu \mathrm{m}$ ) according to Groszman et al. (16). Hepatic venous effluent was collected for 2 min and ${ }^{57} \mathrm{Co}$-radioactivity was determined by gammaspectrometry. The amount of radioactivity injected was determined by counting the syringe before and after injection.

Single-pass extraction of $\left[{ }^{3} \mathrm{H}\right]$ propranolol was measured after injection of $1 \mu \mathrm{Ci}$ of $\left[{ }^{3} \mathrm{H}\right]$ propranolol into the portal vein and collecting effluent for $2 \mathrm{~min}$. Propranolol was extracted from the perfusate into heptane according to Shand et al. (17). Extraction efficiency averaged $99 \pm 1 \%$. Single-pass extraction of $\left[{ }^{14} \mathrm{C}\right]$ antipyrine was determined similarly by injecting $0.2 \mu \mathrm{Ci}$ of $\left[{ }^{14} \mathrm{C}\right]$ antipyrine into the portal vein. Antipyrine was separated from metabolites by high-pressure liquid chromatography according to Eichelbaum with modifications as previously described from this laboratory (18).

In a first set of experiments ( $n=6$ cirrhotics and controls each), verapamil hydrochloride was added to the reservoir in 5-mg increments until portal pressure had been reduced by $25 \%$ in cirrhotic animals. Control livers were paired with a cirrhotic liver and received an equal amoun of verapamil as the cirrhotic liver regardless of its pharmacodynamic effects in the normal liver. After $10 \mathrm{~min}$ of perfusion at the reduced pressure, all measures of viability were repeated. Then, the multiple indicator dilution experiments, shunt fraction, and propranolol and antipyrine extraction measurements were repeated.

To control for potential effects of changes in flow on the different measures of hepatic microcirculation, the effects of mechanically lowering portal pressure were assessed in a second set of five control and cirrhotic rats each. The experimental design was similar: during a control period, all measurements of hepatic microcirculation, function and viability were assessed. Then pressure was lowered by $25 \%$ by closing the valve in the portal inflow (see Fig. 1). After a 10-min adaptation period, all the measures described above were repeated.

Data analysis. The indicator dilution curves were expressed as frequency functions $[h(t)]$, and mean transit times were calculated according to classical indicator dilution theory (19). Vascular space was calculated as the product of flow and $\bar{t}_{\mathrm{RBC}}$; extravascular albumin space (EVA) was calculated as

$\mathrm{EVA}=\operatorname{PF}\left(\bar{t}_{\mathrm{Alb}}-\bar{t}_{\mathrm{RBC}}\right)$, where $\bar{t}_{\mathrm{Alb}}$ and $\bar{t}_{\mathrm{RBC}}$ are the mean transit times for albumin and erythrocytes, respectively, and $P F$ is plasma flow $(19,20)$. The extravascular albumin space thus is model-independent. Extravascular sucrose space was calculated in an analogous fashion, substituting $\bar{t}$ sucrose for $\bar{t}_{\mathrm{Alb}}$. Clearance was calculated as the product of flow determined volumetrically and extraction (21). Means were compared by Student's $t$ test; effects of different treatments within animals were compared with Student's paired $t$ test (22). Linear regression analysis was performed by the method of least squares (22). $P<0.05$ was considered statistically significant.

\section{Results}

During the control period, cirrhotic livers exhibited significantly higher portal resistance and lower liver blood flow expressed on a per gram liver basis than control rats (Table I). Oxygen consumption was significantly lower in cirrhotic livers $(P<0.001$; Table I). In contrast, neither aspartate aminotransferase nor potassium release into the perfusion medium differed significantly between the groups (Table I).

15-30 mg of verapamil were required to lower portal pressure by $25 \%$; taking the volume of the perfusate into account, verapamil concentrations of $8-16 \mathrm{mg} / 100 \mathrm{ml}$ can be calculated. Verapamil administration resulted in a $22 \%$ reduction in hepatic resistance without significant changes in hepatic perfusion in cirrhotic livers. In control livers, portal pressure was reduced by $14 \%$ only; neither flow nor resistance was significantly affected (Table II). In contrast, mechanically lowering portal pressure by a similar amount significantly increased hepatic resistance and consequently resulted in decreased hepatic perfusion in both control and cirrhotic livers (Table II).

Neither verapamil nor mechanical lowering of portal pressure affected viability of the perfused organ as assessed by aspartate aminotransferase or potassium release. Oxygen consumption in control livers did not change after verapamil administration $\left(2.24 \pm 0.08\right.$ vs. $2.14 \pm 0.14 \mu \mathrm{mol} \cdot \mathrm{min}^{-1} \cdot \mathrm{g} \mathrm{liver}^{-1}$; NS) but decreased from $2.28 \pm 0.17$ to $2.05 \pm 0.10 \mu \mathrm{mol} \cdot \mathrm{min}^{-1} \cdot \mathrm{g} \operatorname{liver}^{-1}(P$ $<0.025)$ after mechanically lowering portal pressure. In cirrhotic livers, a similar, although statistically not significant decrease (from $1.78 \pm 0.15$ to $1.44 \pm 0.20$; NS) was seen, whereas verapamil significantly increased oxygen consumption from $1.55 \pm 0.24$ to $1.88 \pm 0.18 \mu \mathrm{mol} \cdot \mathrm{min}^{-1} \cdot \mathrm{g} \mathrm{liver}{ }^{-1}(P<0.02)$.

Intrahepatic shunting averaged $0.10 \pm 0.11 \%$ in control livers; neither pharmacological nor mechanical lowering of portal pressure altered the shunt fraction. Intrahepatic shunting in cirrhotic livers averaged $3.80 \pm 2.85 \%(P<0.005$ compared to con-

Table I. Hemodynamic Features and Viability Criteria of Perfused Cirrhotic and Control Liver

\begin{tabular}{lll}
\hline & $\begin{array}{l}\text { Control } \\
(n=11)\end{array}$ & $\begin{array}{l}\text { Cirrhotic } \\
(n=11)\end{array}$ \\
\hline $\begin{array}{lll}\text { Portal pressure }\left(\mathrm{cmH}_{2} \mathrm{O}\right) \\
\text { Portal flow }\left(\mathrm{ml} \cdot \mathrm{min}^{-1} \cdot \mathrm{g}^{-1}\right)\end{array}$ & $\begin{array}{l}10.4 \pm 0.7 \\
\text { Resistance }\left(\text { dyn } \cdot \mathrm{s} \cdot \mathrm{cm}^{-5} \times 10^{-3}\right)\end{array}$ & $\begin{array}{l}1.48 \pm 0.8 \pm 1.9^{*} \\
\text { Oxygen consumption } \\
\quad\left(\mu \mathrm{mol} \cdot \mathrm{min}^{-1} \cdot \mathrm{g}^{-1}\right)\end{array}$ \\
$\begin{array}{l}\text { Aspartate aminotransferase release } \\
(I U / h)\end{array}$ & $23.1 \pm 5.0$ & $\begin{array}{l}0.99 \pm 0.22^{*} \\
49.3 \pm 15.7^{*}\end{array}$ \\
$\mathrm{~K}^{+}$release $(\mu \mathrm{mol} / \mathrm{h})$ & $4.3 \pm 2.2$ & $1.7 \pm 0.2^{*}$ \\
\hline
\end{tabular}

* $P<0.001$. 
Table II. Hepatic Hemodynamics before and after Lowering Portal Pressure by Verapamil or Mechanically

\begin{tabular}{|c|c|c|c|c|c|c|c|c|c|c|}
\hline & \multirow[b]{2}{*}{$n$} & \multicolumn{3}{|l|}{ Pressure } & \multicolumn{3}{|l|}{ Flow } & \multicolumn{3}{|c|}{ Resistance } \\
\hline & & Before & After & $\Delta$ & Before & After & $\Delta$ & Before & After & $\Delta$ \\
\hline & & $\mathrm{CmH}_{2} \mathrm{O}$ & $\mathrm{CmH}_{2} \mathrm{O}$ & $\mathrm{CmH}_{2} \mathrm{O}$ & \multicolumn{3}{|c|}{$\mathrm{ml} \cdot \mathrm{min}^{-1} \mathrm{~g} \cdot \mathrm{liver} \mathrm{r}^{-1}$} & \multicolumn{3}{|c|}{$d y n \cdot s \cdot \mathrm{cm}^{-5} \times 10^{4}$} \\
\hline Controls (V) & 6 & $10.5 \pm 0.9$ & $8.9 \pm 1.5$ & $-1.6 \pm 1.0 \ddagger$ & $1.3 \pm 0.2$ & $1.1 \pm 0.3$ & $-0.2 \pm 0.3$ & $26 \pm 4$ & $27 \pm 5$ & $1 \pm 6$ \\
\hline Cirrhosis (V) & 6 & $15.2 \pm 1.1$ & $11.7 \pm 1.1$ & $-3.5 \pm 0.8^{11}$ & $0.9 \pm 0.2$ & $0.9 \pm 0.2$ & $-0.0 \pm 0.1$ & $45 \pm 8$ & $35 \pm 7$ & $-10 \pm 4 \S$ \\
\hline Controls (M) & 5 & $10.4 \pm 0.3$ & $7.5 \pm 0.5$ & $-2.9 \pm 0.5^{\prime \prime}$ & $1.7 \pm 0.2$ & $0.9 \pm 0.1$ & $-0.7 \pm 0.1^{11}$ & $20 \pm 3$ & $25 \pm 4$ & $6 \pm 2 \S$ \\
\hline Cirrhosis (M) & 5 & $16.6 \pm 2.1$ & $12.3 \pm 1.8$ & $-4.3 \pm 0.7^{\prime \prime}$ & $1.1 \pm 0.2$ & $0.5 \pm 0.1$ & $-0.5 \pm 0.2^{\| \prime}$ & $55 \pm 18$ & $83 \pm 30$ & $28 \pm 17^{*}$ \\
\hline
\end{tabular}

Abbreviations: V, verapamil; $\mathrm{M}$, mechanical; $\Delta$, difference between before and after values.

$* P<0.02$

$\ddagger P<0.01$

$\S P<0.005$

" $P<0.001$

Statistically significant difference (paired $t$ test) from preceding control period.

trols). In cirrhotic livers verapamil decreased intrahepatic shunting from $4.92 \pm 3.32$ to $3.02 \pm 3.11 \%$ ( $P<0.05$; see Fig. 2$)$, whereas mechanically lowering portal pressure did not affect intrahepatic shunting ( $1.45 \pm 0.99$ vs. $2.36 \pm 1.13 \%$; NS).

Erythrocyte, extravascular albumin, and extravascular sucrose space in control livers averaged $0.180 \pm 0.029,0.077 \pm 0.023$, and $0.097 \pm 0.018 \mathrm{ml} / \mathrm{g}$, respectively. In cirrhotic livers, marked deviation from the flow-limited behavior was observed (Fig. 3). Thus, the albumin curve was almost superimposed upon the erythrocyte curve whereas the sucrose curve increasingly diverged from its associated albumin curve, a pattern suggestive of sinusoidal capillarization $(6,23)$. The erythrocyte space was significantly smaller in cirrhotic livers, averaging $0.148 \pm 0.020$ $\mathrm{ml} / \mathrm{g}(P<0.01)$. Similarly, the extravascular albumin space was reduced to $0.026 \pm 0.013 \mathrm{ml} / \mathrm{g}(P<0.001)$ whereas the extravascular sucrose space was actually increased to $0.184 \pm 0.024$ $\mathrm{ml} / \mathrm{g}(P<0.001)$.

The effects of the different treatments on erythrocyte, albumin, and sucrose space in control and cirrhotic livers are summarized in Table III. Mechanical lowering of portal pressure led to a significant decrease of all three spaces in both control and cirrhotic livers. Verapamil, by contrast, did not significantly affect any of the spaces in control livers. In cirrhotics, it increased extravascular albumin space and did not affect vascular erythrocyte or extravascular sucrose space (Table III). This effect is also illustrated in Figs. 4 and 5, which demonstrate improved

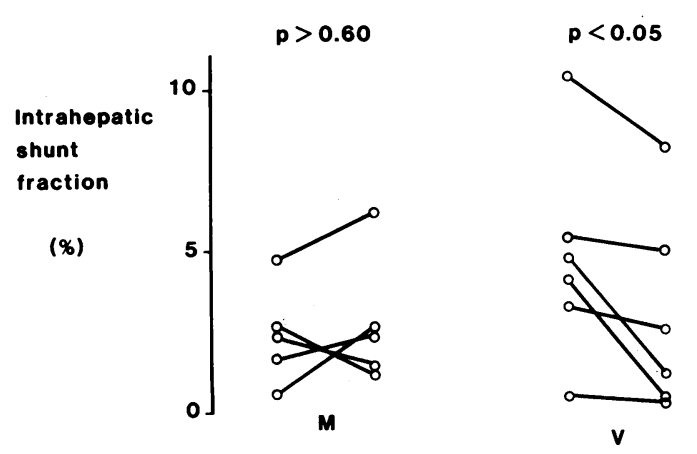

Figure 2. Effects of mechanical $(M)$ and pharmacologic $(V)$ lowering of portal pressure on intrahepatic shunting determined by $15-\mu \mathrm{m}{ }^{57} \mathrm{Co}$ microspheres. Effects of treatment were analyzed by paired $t$ test. exchange of albumin with the extravascular space after verapamil administration. Fig. 5 demonstrates that this effect was due to verapamil and not to decreased pressure and/or flow, in that the extravascular albumin space decreased after mechanical lowering in both control and cirrhotic livers.

Propranolol extraction in controls averaged $97.7 \pm 1.9 \%$; it was significantly reduced in cirrhotics, averaging only $71.3 \pm 11.2 \%(P<0.001)$. Similarly, single-pass extraction of antipyrine was markedly reduced, averaging $22.4 \pm 1.7 \%$ and $4.6 \pm 1.2 \%$ in control and cirrhotic livers, respectively $(P<0.001)$. The effects of the different treatments on drug extraction are summarized in Table IV. Verapamil significantly improved antipyrine but not propranolol extraction in cirrhotics. It had no

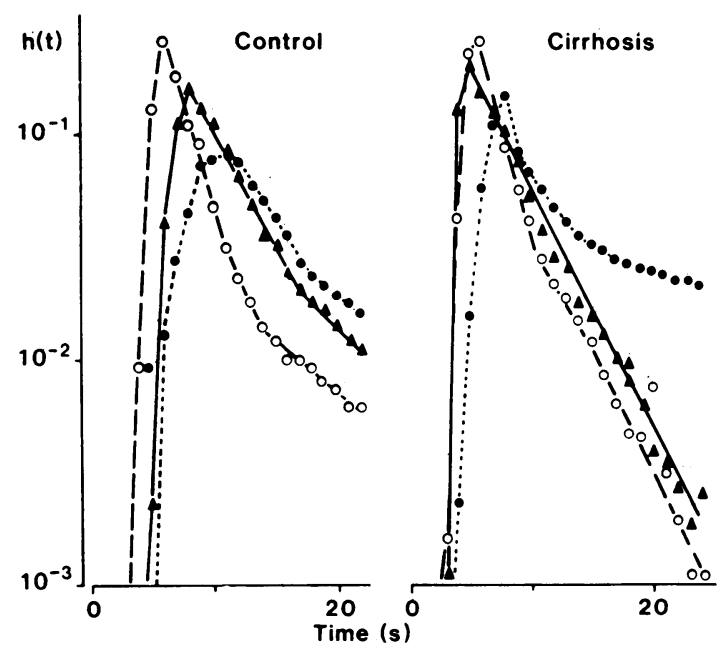

Figure 3. Multiple-indicator dilution curves in normal and cirrhotic liver. The indicators are expressed as frequency function. Erythrocytes (O) appear earlier than albumin ( $\Delta$ ) and sucrose $(\bullet)$ in hepatic venous outflow. The reduction in peak of albumin and sucrose and their delayed appearance are due to the unrestricted exchange of albumin and sucrose with the extravascular space. The control shows the flow-limited pattern typical for liver, whereas in the cirrhotic liver, capillarization has occurred as evidenced by virtual superimposition of the albumin on the erythrocyte curve and diffusion-limited exchange of sucrose as expressed by its biphasic pattern, the first part representing material not having left the sinusoid, and the second part material returning from the extravascular space. 
Table III. Effect of Lowering Portal Pressure with Verapamil or Mechanically on Hepatic Vascular and Extravascular Spaces

\begin{tabular}{|c|c|c|c|c|c|c|}
\hline & \multirow{2}{*}{\multicolumn{2}{|c|}{$\begin{array}{l}\text { Vascular } \\
\text { Erythrocytes }\end{array}$}} & \multicolumn{4}{|l|}{ Extravascular } \\
\hline & & & \multicolumn{2}{|l|}{ Albumin } & \multicolumn{2}{|l|}{ Sucrose } \\
\hline & Before & After & Before & After & Before & After \\
\hline & $\mathrm{ml} / \mathrm{g}$ & $\mathrm{ml} / \mathrm{g}$ & $\mathrm{ml} / \mathrm{g}$ & $\mathrm{ml} / \mathrm{g}$ & $\mathrm{ml} / \mathrm{g}$ & $\mathrm{ml} / \mathrm{g}$ \\
\hline Control (V) & $0.158 \pm 0.019$ & $0.141 \pm 0.013$ & $0.073 \pm 0.027$ & $0.053 \pm 0.011$ & $0.087 \pm 0.017$ & $0.073 \pm 0.021$ \\
\hline Cirrhosis (V) & $0.142 \pm 0.023$ & $0.136 \pm 0.027$ & $0.026 \pm 0.013$ & $0.042 \pm 0.016 \ddagger$ & $0.189 \pm 0.021$ & $0.187 \pm 0.032$ \\
\hline Control (M) & $0.206 \pm 0.010$ & $0.169 \pm 0.014 \S$ & $0.082 \pm 0.015$ & $0.045 \pm 0.012^{*}$ & $0.107 \pm 0.013$ & $0.067 \pm 0.007 \ddagger$ \\
\hline Cirrhosis (M) & $0.154 \pm 0.011$ & $0.098 \pm 0.021^{*}$ & $0.027 \pm 0.012$ & $0.005 \pm 0.002 \ddagger$ & $0.178 \pm 0.023$ & $0.114 \pm 0.025 \ddagger$ \\
\hline
\end{tabular}

Abbreviations: V, verapamil; $\mathrm{M}$, mechanical.

$* P<0.05$

$\ddagger P<0.025\}$ Statistically significant difference from preceding control period (before) by paired $t$ test.

$\S P<0.05\}$

effect in control livers. Mechanical lowering of portal pressure did not affect drug extraction in either group (Table IV). The effects of lowering portal pressure on drug extraction are shown graphically in Fig. 6.

When clearance was calculated as the product of flow (Table I) and drug extraction (Table IV), marked differences were apparent again. Thus, antipyrine clearance averaged $0.332 \pm 0.069$ and $0.046 \pm 0.018 \mathrm{ml} \cdot \mathrm{min}^{-1} \cdot \mathrm{g}^{-1}(P<0.001)$ in controls and cirrhotics, respectively. The corresponding propranolol clearances were $1.45 \pm 0.28$ and $0.70 \pm 0.19 \mathrm{ml} \cdot \mathrm{min}^{-1} \cdot \mathrm{g}^{-1}(P<0.001)$. Antipyrine and propranolol clearance correlated significantly in both control $(r=0.938 ; P<0.001)$ and cirrhotic $(r=0.679 ; P$ $<0.05$ ) rats, but the two regression lines differed obviously (Fig. 7). Because mechanically lowering the portal pressure had effects on blood flow that were different from those of verapamil (Table

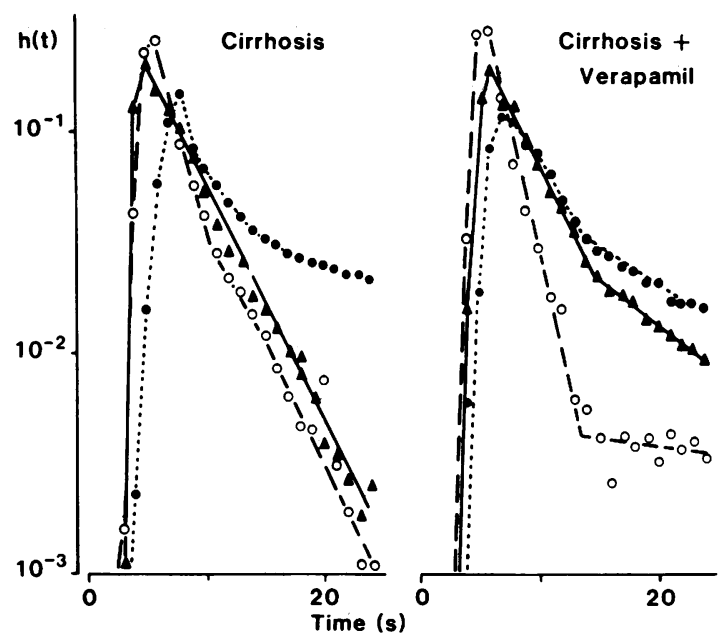

Figure 4. Multiple-indicator dilution curves in a cirrhotic liver before and after verapamil administration. Erythrocyte (O), albumin ( $\Delta$ ), and sucrose ( $\bullet$ ) curves are shown. Evidence of capillarization can be seen in both set of curves. It is evident, however, that exchange of albumin and of sucrose was improved after verapamil administration. The break in the monoexponential decay of the indicators after verapamil administration was seen in four of six livers. It probably reflects perfusion of sinusoids with slower transit times and better exchange characteristics for albumin.
II), the effects of the two manipulations on drug clearance were more marked than those on drug extraction. Mechanically lowering portal pressure decreased the clearance of both drugs in control as well as in cirrhotic livers, whereas verapamil had no effect in control livers (Table V). In cirrhotic livers, by contrast, verapamil significantly improved antipyrine, but not propranolol clearance (Table V).

Neither shunted fraction, portal pressure, portal flow, nor hepatic resistance correlated with either drug clearance (data not shown). By contrast, both antipyrine (Fig. 8) and propranolol (Fig. 9) clearance correlated significantly with extravascular albumin space in cirrhotic livers. No such correlation existed for either drug in controls (Figs. 8 and 9).

\section{Discussion}

This study demonstrates that, in a rat model of portal hypertension, verapamil is able to $(a)$ reduce portal vascular resistance, (b) to decrease intrahepatic shunting, $(c)$ to increase the extravascular space accessible to albumin, and $(d)$ to increase oxygen

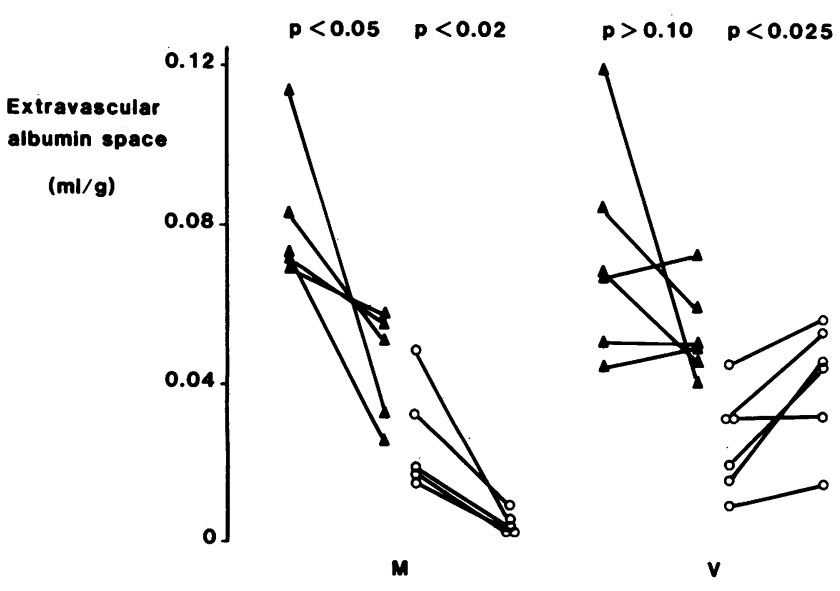

Figure 5. Effects of mechanical $(M)$ and pharmacologic $(V)$ lowering of portal pressure in control $(\Lambda)$ and cirrhotic $(0)$ animals on extravascular albumin space. Extravascular albumin space was determined from ${ }^{51} \mathrm{Cr}$-erythrocyte and ${ }^{125} \mathrm{I}$-albumin dilution curves. Effects of treatments were analyzed by paired $t$ test. 
Table IV. Effects of Mechanically and Pharmacologically

Lowering Portal Pressure on Single-pass Extraction of Antipyrine and Propranolol

\begin{tabular}{lrrlll}
\hline & \multicolumn{2}{l}{ Antipyrine } & & & Propranolol \\
\cline { 2 - 3 } \cline { 5 - 6 } & \multicolumn{1}{l}{ Before } & \multicolumn{1}{c}{ After } & & Before & After \\
\hline & $\%$ & $\%$ & $\%$ & $\%$ \\
Control (M) & $22.0 \pm 2.3$ & $21.0 \pm 2.3$ & & $97.9 \pm 1.8$ & $97.5 \pm 2.1$ \\
Cirrhosis (M) & $4.7 \pm 1.1$ & $4.6 \pm 0.9$ & & $69.4 \pm 7.4$ & $71.7 \pm 5.9$ \\
Control (V) & $22.7 \pm 1.1$ & $22.9 \pm 1.4$ & & $97.4 \pm 2.1$ & $99.1 \pm 0.3$ \\
Cirrhosis (V) & $4.5 \pm 1.4$ & $5.3 \pm 1.3^{*}$ & & $73.0 \pm 14.1$ & $79.2 \pm 8.4$
\end{tabular}

Abbreviations: $\mathbf{M}$, mechanical; $\mathrm{V}$, verapamil.

* $P<0.05$; statistically different from preceding control period (paired $t$ test).

consumption and to improve organ function as assessed by antipyrine clearance. These effects are related to verapamil and not to a reduction in pressure per se, because lowering portal pressure hydrodynamically had either no or adverse effects ( $\mathrm{Ta}$ bles 2-4, Figs. 2, 5, and 6). An attempt to determine whether these beneficial effects were specific for verapamil failed because propranolol had no effects in the perfused rat liver (J. Reichen, unpublished observations). We ascribe these effects of verapamil to a redistribution of flow to sinusoids with different physical characteristics as schematically illustrated in Fig. 10.

Portal hypertension, as measured in vivo, was observed in all cirrhotic animals. The merits and deficiencies of the phenobarbital/carbon tetrachloride model (13) have recently been reviewed (24). Hemodynamically, our cirrhotic livers were characterized by increased portal resistance resulting in decreased portal flow when perfused at the portal pressure determined in vivo (Table 1). In this respect our results are comparable to the study by Varin and Huet (23) who used the same model of cirrhosis induction but at variance with in vivo studies by Vorobioff et al. (25). The latter difference is most likely due to the more advanced nature of the lesion in our study; thus in the present study portal pressure averaged $15.8 \mathrm{cmH}_{2} \mathrm{O}$, as compared with $13.5 \mathrm{cmH}_{2} \mathrm{O}$ in Vorobioff's study, and $73 \%$ of our animals as compared with $50 \%$ of theirs had ascites.
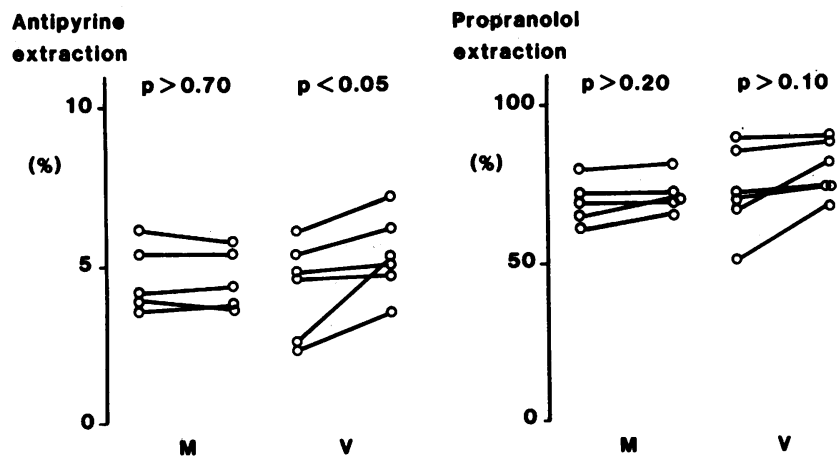

Figure 6. Effects of mechanical $(M)$ and pharmacologic $(V)$ lowering portal pressure on antipyrine and propranolol extraction in cirrhotic livers. Verapamil significantly improved antipyrine extraction only. It did not affect propranolol extraction. Mechanically lowering portal pressure did not affect extraction of either drug.

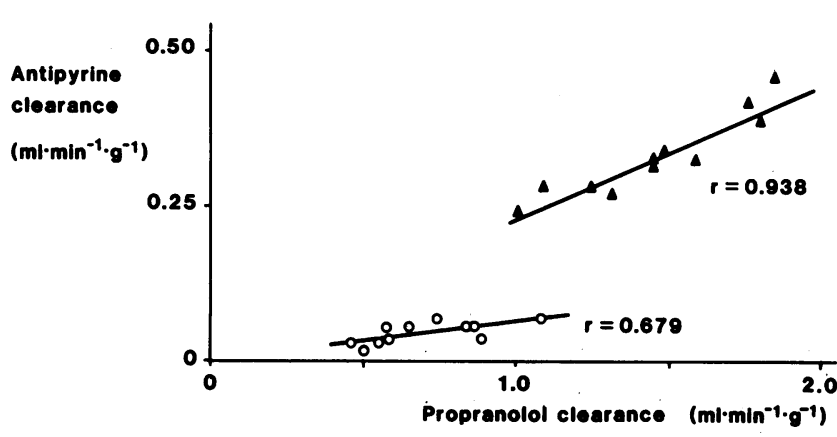

Figure 7. Relationship between antipyrine and propranolol clearance in control ( $\Delta)$ and cirrhotic livers (0). The corresponding regression equations were $y=0.001+0.228 \cdot x$ and $y=0.003+0.062 \cdot x$.

Anatomic intrahepatic shunts, defined as the fraction of 15$\mu \mathrm{m}$ microspheres passing through the liver, was quantitatively not very important, averaging only $3.8 \%$ (range $0.9-10 \%$ ). This figure is in the range of those published for the perfused liver (23) but lower than that reported from several in vivo studies $(25,26)$.

Multiple-indicator dilution studies showed a transition from the usual, flow-limited behavior of albumin and sucrose to diffusion-limited exchange (27). This can be qualitatively inferred from the shape of the indicator dilution curves: flow-limited exchange has been postulated by Goresky $(20,27)$ to result in a smooth decay of intra- and extravascular reference substances, the latter being reduced in magnitude (by a factor depending on the ratio of intra/extravascular volume of distribution) and delayed (by time spent in large, nonexchanging vessels). Diffusionlimited exchange, by contrast, occurs in tight capillaries such as those of the myocardium (27). It is characterized by the albumin curve's being almost superimposed upon the erythrocyte curve and by diffusible reference substances, such as sucrose, that show a deviation from the behavior predicted from the albumin curve owing to diffusion across the endothelial barrier (27). This results in a biphasic curve, the first part representing material that has not left the intravascular plasma space, and the second one material returning from the extravascular sucrose space (27).

Similar distortions of multiple indicator dilution curves in humans and rats have been ascribed to sinusoidal capillarization $(6,23)$. Such capillarization has been demonstrated by trans-

Table V. Effects of Mechanical and Pharmacologic Lowering of Portal Pressure on Antipyrine and Propranolol Clearance

\begin{tabular}{llllll}
\hline & \multicolumn{2}{l}{ Antipyrine clearance } & & \multicolumn{2}{l}{ Propranolol clearance } \\
\cline { 2 - 3 } \cline { 5 - 6 } \cline { 5 - 6 } & Before & After & & Before & After \\
\hline & $m l / g$ & $m l / g$ & $m l / g$ & $m l / g$ \\
Control (M) & $0.37 \pm 0.08$ & $0.20 \pm 0.05^{*}$ & & $1.66 \pm 0.22$ & $0.92 \pm 0.17^{*}$ \\
Cirrhosis (M) & $0.05 \pm 0.02$ & $0.03 \pm 0.01 \ddagger$ & & $0.76 \pm 0.21$ & $0.38 \pm 0.10 \ddagger$ \\
Control (V) & $0.30 \pm 0.04$ & $0.25 \pm 0.07$ & & $1.28 \pm 0.20$ & $1.09 \pm 0.29$ \\
Cirrhosis (V) & $0.04 \pm 0.02$ & $0.05 \pm 0.02 \S$ & & $0.66 \pm 0.18$ & $0.71 \pm 0.16$
\end{tabular}

Abbreviations: $M$, mechanical; $V$, verapamil.

$* P<0.001$

$\ddagger P<0.02$

$\S P<0.01\}$ period (paired $t$ test) 


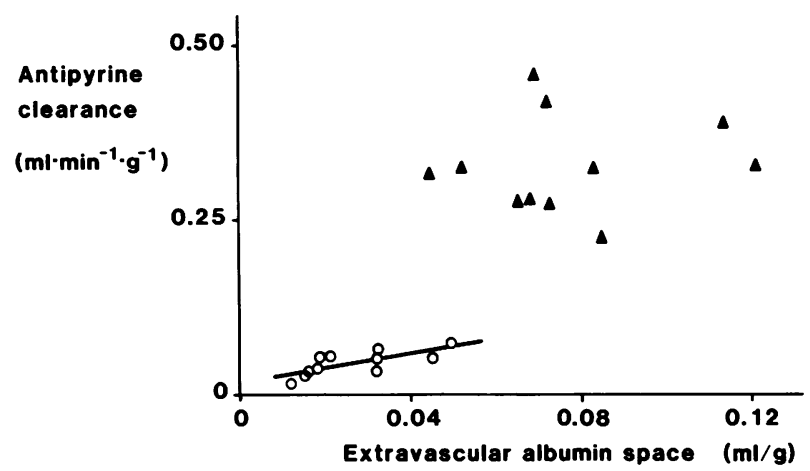

Figure 8. Relationship between antipyrine clearance and extravascular albumin space in control ( $\mathbf{(})$ and cirrhotic (O) livers. No statistically significant correlation existed in control animals $(r=0.114)$. In cirrhotics, the regression equation was $y=0.015+0.669 \cdot x(r=0.766$; $P<0.01$ )

mission electron microscopy $(4,5)$ and basement membranelike deposits have been demonstrated immunohistochemically (28, 29). The functional significance of these changes in cirrhosis have first been appreciated by Huet and colleagues $(6,23)$, who demonstrated a close correlation between changes in function and sinusoidal capillarization. We found a similar correlation between the clearance of both a high (propranolol, Fig. 9) and a low (antipyrine, Fig. 8) extraction compound and extravascular albumin space. No other parameter of deranged hepatic hemodynamics (flow, resistance, portal pressure, intrahepatic shunting) correlated with function in our study. The decrease in extravascular albumin space is comparable to that described by Varin and Huet (23); similarly, the increase in extravascular sucrose space is comparable to values reported in the literature. This increase in sucrose space has been ascribed tentatively to the ability of sucrose to permeate into fibrous tissue in the extravascular space (23).

Hepatic function was markedly reduced measured by the clearance of both, a low and a high extraction compound, namely antipyrine and propranolol, respectively. This is in accordance with findings in humans $(30-32)$ and rats $(26,33)$. Presumably, the reduced oxygen consumption is also an expression of decreased function rather than of compromised viability of the organ. Viability of perfused cirrhotic livers was comparable to control livers when transaminase and potassium release are con-

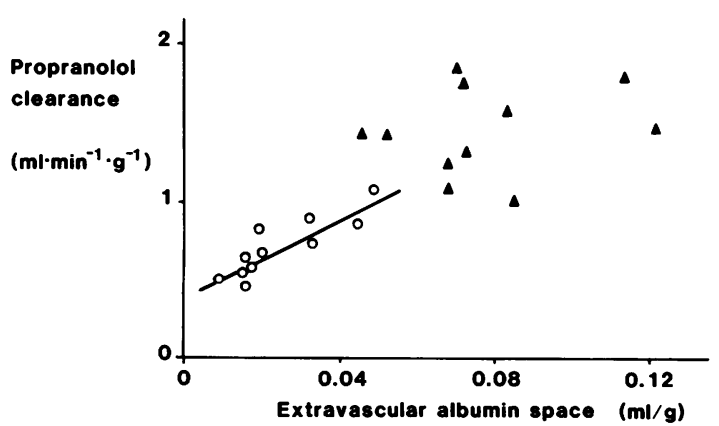

Figure 9. Relationship between propranolol clearance and extravascular albumin space in control ( $\Delta$ ) and cirrhotic $(0)$ animals. No statistically significant correlation existed in control animals $(r=0.185)$. In cirrhotics, the regression equation was $y=0.30+8.11 \cdot x(r=0.846$; $P<0.01$ ).

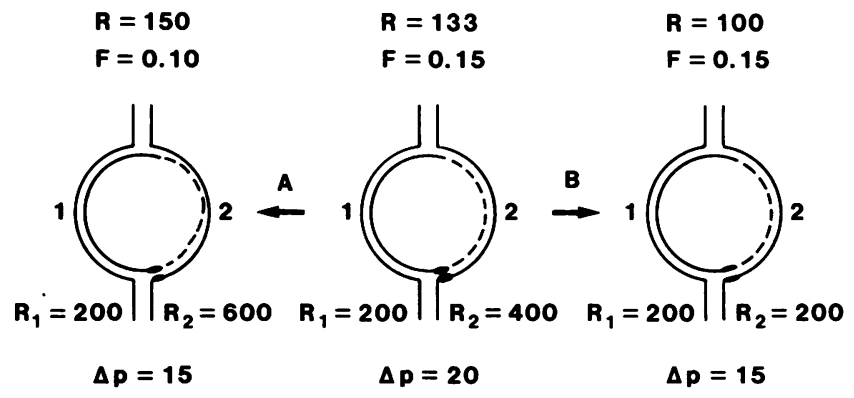

Figure 10. Schematic representation of the potential effects of mechanically $(A)$ or pharmacologically $(B)$ lowering portal pressure. The liver is represented by a capillarized sinusoid $(I)$ and a sinusoid with near-normal exchange characteristics (2). Total flow in parallel circuits is $F=\Delta P / R_{1}+\Delta P / R_{2}$ and total resistance can be calculated from the conductances $(1 / R)$ as $1 / R=1 / R_{1}+1 / R_{2}=F / \Delta P$. Verapamil could act by relaxing endothelial cells at sinusoids with better exchange characteristics $(B): R_{2}$ diminishes, leading to reduced total resistance, reduced pressure, and constant flow. Mechanically lowering portal pressure, by contrast, could lead to collapse of some sinusoids, further increasing their resistance: flow decreases and total resistance increases $(A)$.

sidered (Table I). Reduced oxygen consumption was reported in a similar study in the rat (23) and demonstrated in end-stage cirrhosis in humans (34). We have recently described reduced oxygen consumption in hepatocytes isolated from cirrhotic livers where hemodynamic alterations are excluded (35).

Verapamil significantly reduced portal pressure by reducing portal vascular resistance, leaving portal flow unaffected (Table II). This apparent paradox is easily explained by assuming perfusion of sinusoids with different physical characteristics (see Fig. 10). Verapamil could exert these effects by acting on endothelial cells, the main determinants of sinusoidal perfusion $(36,37)$. Verapamil is known to affect all smooth muscle cells (9), including those of rat portal vein (38); it is unknown, however, whether verapamil affects hepatic endothelial cells. The verapamil concentrations achieved in our experiments can be calculated to be $8-16 \mathrm{mg} / 100 \mathrm{ml}$ or $200-400 \mu \mathrm{mol} / \mathrm{liter}$. This is well above the concentration where verapamil changes from a specific calcium antagonist to a sodium channel blocker (39). Thus, we cannot exclude that nonspecific membrane effects of verapamil were contributing to our results.

Inspecting the effects of verapamil on the indicator dilution curves (Fig. 4), it appears unlikely that these effects were achieved by regulation of large vessels. Similarly, an alteration of sinusoidal capillarization in a given sinusoid appears rather unlikely, inasmuch as this consists of formation of a basement membranelike structure occluding the fenestrations in the sinusoidal lining cells (4-6). Another possibility is that verapamil alters the size and/ or number of endothelial fenestrations. Whether dynamic alteration of these important determinants of transsinusoidal exchange occurs is unknown. They are known, however, to be altered in response to increased portal pressure in the rat (40) and in alcohol-induced chronic liver injury in baboons (41). Considering the nature of capillarization (4-6), however, such a mechanism appears rather unlikely also. Again, the mechanism invoked to explain the altered hemodynamics after verapamil administration, namely perfusion of sinusoids with different physical characteristics (Fig. 10), appears most apt to explain the observed changes in sinusoidal extravascular space. The per- 
fusion of sinusoids with different accessibility of albumin to the extravascular space could also explain the observed improvement in oxygen consumption and antipyrine clearance.

A similar mechanism can be invoked to explain the decrease in extravascular albumin space, impaired oxygen consumption, and decreased antipyrine clearance observed after hydrodynamic lowering of portal pressure: a decrease in pressure could lead to partial collapse of sinusoids with relatively well-maintained exchange characteristics (Fig. 10). Such an explanation could also explain the failure of extraction to increase when flow decreases as predicted from theoretical considerations (21). The effects achieved with verapamil and hydrodynamic alterations of portal pressure differ from increasing pressure pharmacologically with norepinephrine (42) or hydrodynamically by venous outflow obstruction (43). Increasing the pressure leads to alteration of vascular, but not extravascular volumes in normal liver (42). We ascribe these effects to the mechanisms invoked in Fig. 10: in our experiments we speculate to have altered distribution of perfusion to sinusoids rather than perfusion of a single sinusoid. The variations induced by the different manipulations were not sufficient, however, to systematically explore the relation between flow and the different vascular and extravascular spaces in cirrhotic liver.

At present we can only speculate why antipyrine but not propranolol clearance was affected by verapamil. We tentatively ascribe the difference to the different lipid solubility of antipyrine and propanolol. Nonspecific tissue binding, presumably in a lipid compartment, is the main determinant of first-pass uptake of propranolol $(44,45)$ entry into cells occurring very rapidly by passive diffusion (45). Metabolism, leading to definitive clearance from the liver, occurs much more slowly (45). By contrast, antipyrine clearance depends entirely on metabolism by the monooxygenase system $(46,47)$ and is independent of liver blood flow (48). Thus, it is conceivable that, in measuring singlepass uptake of propranolol, we measured the passive distribution phenomena only, and that prolonged observation of the fate of propranolol would reveal effects similar to those achieved for antipyrine. Alternatively, the different effects could be due to different protein binding, antipyrine being much less extensively bound (46) than propranolol (44).

Pharmacologic therapy of portal hypertension has been used in clinical medicine in the acute treatment of variceal hemorrhage by vasopressin and somatostatin $(1,49)$. Both act by decreasing splanchnic flow resulting in lowered hepatic oxygen tension and further reduction in the function of an already compromised organ (49). Such adverse effects can be partially overcome by combination with vasodilators such as glyceryl trinitrate (50) or nitroprusside (51).

Chronic pharmacotherapy of portal hypertension has similarly been achieved by reducing splanchnic blood flow by beta blockade. Thus, Lebrec and colleagues could demonstrate reduction in esophageal variceal bleeding (2), and improvement in survival (3) in patients treated with propranolol. This was not associated with a reduction in hepatic function in the patients of Lebree et al. (52). However, several reports of adverse effects of propranolol therapy have appeared; these have recently been editorially reviewed (53). In patients with more advanced liver disease, propranolol has not been effective (54). This could be due to increased hepatic vascular resistance (55) and/or reduced sensitivity to norepinephrine in the splanchnic vascular bed maintaining high flow against an unchanged hepatic resistance (56). In our model, propranolol even at very high doses up to
$200 \mathrm{mg}$, had no effect on any of the parameters measured (J. Reichen, unpublished observations); this is in agreement with data obtained in the intact animal (55).

In conclusion, we have confirmed that sinusoidal capillarization, as measured by reduction in extravascular space available to albumin, is a major determinant of function of the cirrhotic liver. Hepatic resistance in an animal model of liver cirrhosis is not fixed but can be pharmacologically influenced, e.g., with calcium channel blocking drugs such as verapamil. The doses required to achieve this, however, are rather high. Reduction of hepatic vascular resistance could be due to an effect on endothelial cells. We propose that this leads to a redistribution of flow to areas with an extravascular space more accessible to albumin, decrease in intrahepatic shunting, improvement in oxygen consumption, and partial restoration of impaired hepatic function. Whether our findings can be extrapolated to humans remains to be proven. Recent demonstration of verapamil effects on portal pressure (11) and hepatic function (12) are encouraging.

\section{Acknowledgments}

The technical assistance of Mr. K. Gosselin, the artwork by Ms. M. Kappeler, and the secretarial assistance of Ms. R. Steiner are gratefully acknowledged.

Dr. Reichen was the recipient of a Research Career Development Award of the National Institutes of Health (K04 AM-1189) and of the Swiss National Foundation for Scientific Research (3.731.82). This study was supported by grant AM-32511 from the National Institutes of Health and grant 3.823.0.84 from the Swiss National Foundation.

\section{References}

1. Bosch, J., D. Kravetz, and J. Rodes. 1981. Effects of somatostatin in hepatic and systemic hemodynamics in patients with cirrhosis of the liver: comparison with vasopressin. Gastroenterology. 80:1157-1175.

2. Lebrec, D., T. Poynard, P. Hillon, and J. P. Benhamou. 1981. Propranolol for prevention of recurrent gastrointestinal bleeding in patients with cirrhosis. N. Engl. J. Med. 305:1371-1374.

3. Lebrec, D., T. Poynard, J. Bernuau, E. Bercoff, O. Nouel, J. P. Capron, R. Poupon, M. Bouvry, B. Rueff, and J. P. Benhamou. 1984. A randomized controlled study of propranolol for prevention of recurrent gastrointestinal bleeding in patients with cirrhosis: a final report. $\mathrm{He}$ patology. 4:355-358.

4. Popper, H., H. Elias, and D. E. Petty. 1952. Vascular pattern of cirrhotic liver. Am. J. Clin. Pathol. 22:717-729.

5. Schaffner, F., and H. Popper. 1963. Capillarization of hepatic sinusoids in man. Gastroenterology. 44:239-242.

6. Huet, P. M., C. A. Goresky, J. P. Villeneuve, D. Marleau, and J. O. Lough. 1982. Assessment of liver microcirculation in human cirrhosis. J. Clin. Invest. 70:1234-1244.

7. Rappaport, A. M., P. J. MacPhee, M. M. Fisher, and M. J. Phillips. 1983. The scarring of the liver acini (cirrhosis): tridimensional and microcirculatory considerations. Virchows Arch A Pathol. Anat. Histopathol. 402:107-137.

8. Hamann, S. R., R. A. Blouin, and R. G. McAllister. 1984. Clinical pharmacokinetics of verapamil. Clin. Pharmacokinet. 9:26-41.

9. Karaki, H., and G. B. Weiss. 1984. Calcium channels in smooth muscle. Gastroenterology. 87:960-970.

10. Meredith, P. A., H. L. Elliot, F. Pasanisi, A. W. Kelman, D. J. Sumner, and J. L. Reid. 1985. Verapamil pharmacokinetics and apparent hepatic and renal blood flow. Br. J. Clin. Pharmacol. 20:101-106.

11. Freeman, J. G., J. R. Barton, and C. O. Record. 1985. Effect of isosorbide dinitrate, verapamil, and labetalol on portal pressure in cirrhosis. Br. Med. J. 291:561-562.

12. Miotti, T., and J. Reichen. 1985. Verapamil improves fractional clearance of indocyanine green in patients with liver cirrhosis. J. Hepatol. 2(Suppl.):S291. (Abstr.) 
13. McLean, E. K., A. E. M. McLean, and P. M. Sutton. 1969. Instant cirrhosis. An improved method for producing cirrhosis of the liver in rats by simultaneous administration of carbon tetrachloride and phenobarbitone. Br. J. Exp. Pathol. 50:502-506.

14. Reichen, J., and G. Paumgartner. 1976. Uptake of bile acids by the perfused rat liver. Am. J. Physiol. 231:734-742.

15. Reichen, J., and M. Le. 1983. Taurocholate, but not taurodehydrocholate, increases biliary permeability to sucrose. Am. J. Physiol. 245:G651-G655.

16. Groszman, R. J., J. Vorobioff, and E. Riley. 1982. Splanchnic hemodynamics in portal-hypertensive rats: measurement with gammalabeled microspheres. Am. J. Physiol. 242:G156-G160.

17. Shand, D. G., E. M. Nuckolls, and J. A. Oates. 1970. Plasma propranolol levels in adults: with observations in four children. Clin. Pharmacol. Ther. 11:112-120.

18. Berry, W. R., G. Kirshenbaum, C. Hoilien, M. Le, and J. Reichen. 1985. Taurolithocholate increases heme catabolism and alters the clearance of antipyrine in the rat. Gastroenterology. 88:397-402.

19. Meier, P., and K. L. Zierler. 1954. On the theory of the indicator dilution method for measurement of blood flow and volume. J. Appl. Physiol. 6:731-744.

20. Goresky, C. A. 1963. A linear method for determining liver sinusoidal and extravascular volumes. Am. J. Physiol. 204:626-640.

21. Rowland, M., L. Z. Benet, and G. G. Graham. 1973. Clearance concepts in pharmacokinetics. J. Pharmacokinet. Biopharm. 1:123-136.

22. Snedecor, G. W., and W. G. Cochran. 1967. Statistical Methods. Iowa State University Press, Ames, Iowa.

23. Varin, R., and P. M. Huet. 1985. Hepatic microcirculation in the perfused cirrhotic rat liver. J. Clin. Invest. 76:1904-1912.

24. Tamayo, R. P. 1983. Is cirrhosis of the liver experimentally produced by $\mathrm{CCl}_{4}$ an adequate model of human cirrhosis? Hepatology. 3: 112-120.

25. Vorobioff, J., J. E. Bredfeldt, and R. J. Groszman. 1984. Increased blood flow through the portal system in cirrhotic rats. Gastroenterology. 87:1120-1126.

26. Wood, A. J. J., J. P. Villeneuve, R. A. Branch, L. W. Rogers, and D. G. Shand. 1979. Intact hepatocyte theory of impaired drug metabolism in experimental cirrhosis in the rat. Gastroenterology. 76:13581362.

27. Goresky, C. A., W. H. Ziegler, and G. G. Bach. 1970. Capillary exchange modelling. Barrier-limited and flow-limited distribution. Circ. Res. 27:739-764.

28. Hahn, E., G. Wick, D. Pencev, and R. Timpl. 1980. Distribution of basement membrane proteins in normal and fibrotic human liver: collagen type IV, laminin, and fibronectin. Gut. 21:63-71.

29. Bianchi, F. B., G. Biagini, C. Ballardini, A. Faccani, E. Pisi, R. Laschi, L. Liotta, and S. Garbisa. 1984. Basement membrane production by hepatocytes in chronic liver disease. Hepatology. 4:1167-1172.

30. Branch, R. A., J. A. James, and A. E. Read. 1976. The clearance of antipyrine and indocyanine green in normal subjects and in patients with chronic liver disease. Clin. Pharmacol. Ther. 20:81-89.

31. Pessayre, D., D. Lebrec, V. Descatoire, M. Peignoux, and J. P. Benhamou. 1978. Mechanism for reduced drug clearance in patients with cirrhosis. Gastroenterology. 74:566-571.

32. Huet, P. M., and J. P. Villeneuve. 1983. Determinants of drug disposition in patients with cirrhosis. Hepatology. 3:913-918.

33. Villeneuve, J. P., A. J. J. Wood, D. G. Shand, L. Rogers, and R. A. Branch. 1978. Impaired drug metabolism in experimental cirrhosis in the rat. Biochem. Pharmacol. 27:2577-2581.

34. Loda, M., G. H. A. Clowes, A. Nespoli, L. Bigatello, D. H. Birkett, and J. O. Menzoian. 1984. Encephalopathy, oxygen consumption, visceral amino acid clearance, and mortality in cirrhotic surgical patients. $\mathrm{Am}$. J. Surg. 147:542-550.

35. Hoilien, C., W. Berry, M. Le, and J. Reichen. 1984. Decreased uptake of taurocholate and ouabain by cirrhotic rat hepatocytes: evidence for the sick cell hypothesis. Hepatology. 4:750. (Abstr.)

36. Reilly, F. D., R. V. W. Dimlich, E. V. Cilento, and R. S. McCuskey. 1983. Hepatic microvascular regulatory mechanisms. III.
Aminergic mechanisms related to mast cells. Int. J. Microcirc. Clin. Exp. 2:61-73.

37. Rappaport, A. M. 1979. Hepatic blood flow: morphologic aspects and physiologic regulation. Int. Rev. Physiol. 21:1-63.

38. Jetley, M., and A. H. Weston. 1980. Some effects of sodium nitroprusside, methoxyverapamil (D600) and nifedipine on rat portal vein. Br. J. Pharmacol. 68:311-320.

39. Norris, D. K., and H. P. Bradford. 1985. On the specificity of verapamil as a calcium channel blocker. Biochem. Pharmacol. 34:19531956.

40. Fraser, R., L. M. Bowler, W. A. Day, D. Bobbs, H. D. Johnson, and $D$. Lee. 1980. High perfusion pressure damages the sieving ability of sinusoidal endothelium in rat liver. Br. J. Exp. Pathol. 61:222-228.

41. Mak, K. M., and C. S. Lieber. 1984. Alterations in endothelial fenestrations in liver sinusoids of baboons fed alcohol: a scanning electron microscopic study. Hepatology. 4:386-391.

42. Greenway, C. V., K. L. Seaman, and I. R. Innes. 1985. Norepinephrine on venous compliance and unstressed volume in cat liver. Am. J. Physiol. 248:H468-H476.

43. Cousineau, D., C. A. Goresky, C. P. Rose, and S. Lee. 1985. Reflex sympathetic effects on liver vascular space and liver perfusion in dogs. Am. J. Physiol. 248:H186-H192.

44. Evans, G. H., G. R. Wilkinson, and D. G. Shand. 1973. The disposition of propranolol. IV. A dominant role for tissue uptake in the dose-dependent extraction of propranolol by the perfused rat liver. $J$. Pharm. Exp. Ther. 186:447-454.

45. Ong, H., P. Du Souich, and C. Marchand. 1981. In vivo study of propranolol and metabolite(s) disposition in rat liver. Drug Metab. Dispos. 9:529-534.

46. Brodie, B. B., and J. Axelrod. 1950. The fate of antipyrine in man. J. Pharmacol. Exp. Ther. 98:97-104.

47. Chang, S. L., S. D. Nelson, and R. H. Levy. 1984. Correlation between antipyrine clearance and cytochrome $\mathrm{P450}$ after phenobarbital induction in rat. Drug Metab. Dispos. 12:139-141.

48. Branch, R. A., D. G. Shand, G. R. Wilkinson, and A. S. Nies. 1974. Increased clearance of antipyrine and d-propranolol after phenobarbital treatment in the monkey: relative contributions of enzyme induction and increased hepatic blood flow. J. Clin. Invest. 53:11011107.

49. Barbare, J. C., R. Poupon, P. Jaillon, P. Bories, M. Aussonaire, F. Dorais, H. Michel, and G. Cheymol. 1984. The influence of vasoactive agents on metabolic activity of the liver in cirrhosis: a study of the effects of posterior pituitary extract, vasopressin and somatostatin. Hepatology. 4:59-62.

50. Groszman, R. J., D. Kravetz, J. Bosch, M. Glickman, J. Bruix, J. Bredtfeldt, H. O. Conn, J. Rodes, and E. H. Stoerer. 1982. Nitroglycerin improves the hemodynamic response to vasopressin in portal hypertension. Hepatology. 2:757-762.

51. Mols, P., R. Hallemans, M. Van Kuyk, C. Melot, P. Lejeune, H. Ham, F. Vertongen, and R. Naeije. 1984. Hemodynamic effects of vasopressin, alone and in combination with nitroprusside, in patients with liver cirrhosis and portal hypertension. Ann. Surg. 199:176-181.

52. Larrey, D., D. Lebrec, E. Bercoff, and D. Pessayre. 1983. Propranolol does not further decrease the clearance of antipyrine in patients with alcoholic cirrhosis. Clin. Sci. (Lond.). 65:203-205.

53. Conn, H. O. 1984. Propranolol in portal hypertension: problems in paradise? (editorial). Hepatology. 4:560-564.

54. Burroughs, A. K., W. J. Jenkins, S. Sherlock, A. Dunk, R. P. Walt, T. O. K. Osuafor, S. Mackie, and R. Dick. 1983. Controlled trial of propranolol for the prevention of recurrent variceal hemorrhage in patients with cirrhosis. N. Engl. J. Med. 309:1539-1542.

55. Kroeger, R. J., and R. J: Groszman. 1985. Increased portal-venous resistance hinders portal pressure reduction during the administration of beta-adrenergic blocking agents in a portal-hypertensive model. $\mathrm{He}$ patology. 5:97-101.

56. Kiel, J. W., V. Pitts, J. N. Benoit, N. D. Granger, and A. P. Sheperd. 1985. Reduced vascular sensitivity to norepinephrine in portalhypertensive rats. Am. J. Physiol. 248:G192-G195. 